\title{
Mixed product surfaces in euclidean 3-space $\mathbb{E}^{3}$
}

\author{
Betul Bulca and Kadri Arslan \\ Department of Mathematics, Uludağ University, Bursa, Turkey \\ Received: 16 September 2015, Revised: 20 January 2016, Accepted: 27 January 2016 \\ Published online: 20 April 2016.
}

\begin{abstract}
In the present study we define a new kind of product surfaces namely mixed product which are product of plane curve and space curve in 3-dimensional Euclidean space. We give the original results of mixed product surface patches of flat or minimal type in $\mathbb{E}^{3}$. Further, we give some examples of these kind of surfaces and plot their graphics.
\end{abstract}

Keywords: Spherical product, mixed product, surfaces, superquadrics.

\section{Introduction}

The problem of recovering the shape of objects from unstructured $3 \mathrm{D}$ data is important in many areas of computer graphics and computer vision, including robotics, medical images and the automatic construction of virtual environments. In last 30 years, much work has been done focus of finding suitable models for the recovery of objects from 3D data. This work has largely proposed the use of some form of para- metric model, most commonly spherical product of two 2D curves. Quadrics are the simple type of these surfaces. So, superquadrics can be also considered as the spherical products of two 2D curves. In fact, superquadrics are solid models that can have fairly simple parametrization of representing a large variety of standard geometric solids, as well as smooth shapes in between. Petland was first who grasped the potential of the superquadratic models and parametric deformations for modelling natural shapes in the context of computer vision [9]. He offered superquadrics in combination with deformations as a shape vocabulary for this part-level representation.

The superquadrics, which are like phonemes in this description, language, can be deformed by stretching, bending, tapering or twisting, and then combined using Boolean operations to built complex objects ([6], pp. 9). It can be observed that superquadric recovery is being integrated, on the one hand, with segmentation ([10], [4], [7]) and on the other hand, with decision making such as categorization [5]. Superquadrics are the special case of the supershapes, provided by Gielis and et al. [3] that have the advantage of representing polygonal with various symmetries. Recently, in [1] the authors present the original results of spherical product surface patches of flat or minimal type.

In the present study we define a new kind of product surfaces which are product of a plane curve with a space curve in $\mathbb{E}^{3}$ which is called spherical product. Mixed products can be considered as the generalization of spherical products. The rest of the paper is organized as follows: In Section 2 we give necessary definitions and theorems as basic concepts. Section 3 presents the original results of mixed product surface patches of flat or minimal type. Section 4 provides some visualizations of mixed product surface. Finally, section 5 concludes the paper and discussed areas for future work. 


\section{Basic concepts}

Let $M$ be a smooth surface in $\mathbb{E}^{n}$ given with the patch $X(u, v):(u, v) \in D \subset \mathbb{E}^{2}$. The tangent space to $M$ at an arbitrary point $p=X(u, v)$ of $M$ is spanned by $\left\{X_{u}, X_{v}\right\}$. In the chart $(u, v)$ the coefficients of the first fundamental form of $M$ are given by

$$
E=\left\langle X_{u}, X_{u}\right\rangle, F=\left\langle X_{u}, X_{v}\right\rangle, G=\left\langle X_{v}, X_{v}\right\rangle
$$

where $\langle$,$\rangle is the Euclidean inner product. We assume that W^{2}=E G-F^{2} \neq 0$, i.e. the surface patch $X(u, v)$ is regular. For each $p \in M$, consider the decomposition $T_{p} \mathbb{E}^{n}=T_{p} M \oplus T_{p}^{\perp} M$ where $T_{p}^{\perp} M$ is the orthogonal component of $T_{p} M$ in $\mathbb{E}^{n}$. Let $\tilde{\nabla}$ be the Riemannian connection of $\mathbb{E}^{n}$.

Let $\chi(M)$ and $\chi^{\perp}(M)$ be the space of the smooth vector fields tangent to $M$ and the space of the smooth vector fields normal to $M$, respectively. Further, given any local vector fields $X_{i}$ and $X_{j}$ tangent to $M$ one can define the second fundamental map of $M$ by $h: \chi(M) \times \chi(M) \rightarrow \chi^{\perp}(M)$;

$$
h\left(X_{i}, X_{j}\right)=\widetilde{\nabla}_{X_{i}} X_{j}-\nabla_{X_{i}} X_{j} 1 \leq i, j \leq 2 .
$$

where $\widetilde{\nabla}$ is the induced connection. This map is well-defined, symmetric and bilinear.

For any arbitrary orthonormal normal frame field $\left\{N_{1}, N_{2}\right\}$ of $M$, recall the shape operator $A: \chi^{\perp}(M) \times \chi(M) \rightarrow \chi(M)$;

$$
A_{N_{i}} X_{i}=-\left(\widetilde{\nabla}_{X_{i}} N_{i}\right)^{T}, \quad X_{i} \in \chi(M)
$$

This operator is bilinear, self-adjoint and satisfies the following equation:

$$
\left\langle A_{N_{k}} X_{i}, X_{j}\right\rangle=\left\langle h\left(X_{i}, X_{j}\right), N_{k}\right\rangle=c_{i j}^{k}
$$

where $1 \leq i, j \leq 2$ and $1 \leq k \leq n-2$.

The equation (2) is called Gaussian formula, and

$$
h\left(X_{i}, X_{j}\right)=\sum_{k=1}^{2} c_{i j}^{k} N_{k}
$$

where $c_{i j}^{k}$ are the coefficients of the second fundamental form.

Further, the Gaussian curvature and mean curvature vector of a regular patch $X(u, v)$ are given by

$$
K=\frac{1}{W^{2}} \sum_{k=1}^{2}\left(c_{11}^{k} c_{22}^{k}-\left(c_{12}^{k}\right)^{2}\right)
$$

and

$$
\vec{H}=\frac{1}{2 W^{2}} \sum_{k=1}^{2}\left(c_{11}^{k} G+c_{22}^{k} E-2 c_{12}^{k} F\right) N_{k}
$$

respectively.

Recall that a surface $M$ is said to be minimal if its mean curvature vector vanishes identically [2]. 


\section{Mixed product surfaces in $\mathbb{E}^{3}$}

Definition 1. Let $\alpha: I \subset \mathbb{R} \longrightarrow \mathbb{E}^{2}$ be Euclidean plane curve and $\beta: J \subset \mathbb{R} \longrightarrow \mathbb{E}^{3}$ a space curve respectively. Put $\alpha(u)=\left(f_{1}(u), f_{2}(u)\right)$ and $\beta(v)=\left(g_{1}(v), g_{2}(v), g_{3}(v)\right)$. Then we define their mixed product patch by

$$
X=\alpha \otimes \beta: \mathbb{E}^{2} \longrightarrow \mathbb{E}^{3} ; X(u, v)=\left(f_{1}(u) g_{1}(v), f_{1}(u) g_{2}(v), f_{2}(u) g_{3}(v)\right) ;
$$

where $u \in I=\left(u_{0}, u_{1}\right)$ and $v \in J=\left(v_{0}, v_{1}\right)$.

In the following result we give the necessary and sufficient conditions for $M$ to become a surface patch in $\mathbb{E}^{3}$.

Theorem 1. If $\alpha(u)$ and $\beta(v)$ are not straight lines passing through the origin then the surface patch $X(u, v)$ is a regular patch.

Proof. The tangent space of $M$ is spanned by the following partial derivatives

$$
\begin{aligned}
& X_{u}(u, v)=\left(f_{1}(v) g_{1}^{\prime}(u), f_{2}(v) g_{1}^{\prime}(u), f_{3}(v) g_{2}^{\prime}(u)\right), \\
& X_{v}(u, v)=\left(f_{1}^{\prime}(v) g_{1}(u), f_{2}^{\prime}(v) g_{1}(u), f_{3}^{\prime}(v) g_{2}(u)\right) .
\end{aligned}
$$

The surface patch $X(u, v)$ is regular if the area element $W=\left\|X_{u} \times X_{v}\right\|$ is a non-zero function.

Assume that the are element $W$ of $M$ vanishes identically. Then from the wedge product

$$
X_{u} \times X_{v}=\left(f_{1}^{\prime} f_{2} g_{2} g_{3}^{\prime}-f_{1} f_{2}^{\prime} g_{2}^{\prime} g_{3}, f_{1} f_{2}^{\prime} g_{1}^{\prime} g_{3}-f_{1}^{\prime} f_{2} g_{1} g_{3}^{\prime}, f_{1} f_{1}^{\prime} g_{1} g_{2}^{\prime}-f_{1} f_{1}^{\prime} g_{1}^{\prime} g_{2}\right)
$$

we get

$$
\begin{aligned}
& f_{1}^{\prime} f_{2} g_{2} g_{3}^{\prime}-f_{1} f_{2}^{\prime} g_{2}^{\prime} g_{3}=0, \\
& f_{1} f_{2}^{\prime} g_{1}^{\prime} g_{3}-f_{1}^{\prime} f_{2} g_{1} g_{3}^{\prime}=0, \\
& f_{1} f_{1}^{\prime} g_{1} g_{2}^{\prime}-f_{1} f_{1}^{\prime} g_{1}^{\prime} g_{2}=0 .
\end{aligned}
$$

A simple calculation gives that

$$
\begin{aligned}
& \frac{f_{1}^{\prime}}{f_{1}}=\frac{f_{2}^{\prime}}{f_{2}}, \\
& \frac{g_{1}^{\prime}}{g_{1}}=\frac{g_{2}^{\prime}}{g_{2}}=\frac{g_{3}^{\prime}}{g_{3}} .
\end{aligned}
$$

So we deduce from the equation (10) that the curves $\alpha(u)$ and $\beta(v)$ are both straight lines passing through the origin. This contradicts with our hypothesis. So, the area element $W$ of $M$ can not be zero provided that the surface patch $X(u, v)$ is a regular.

We give the following examples of mixed products;

Example 1.The mixed product $\alpha(u)=\left(f_{1}(u), f_{2}(u)\right)$ with $\beta(v)=\left(g_{1}(v), g_{2}(v), 1\right)$ forms the surface patch

$$
X(u, v)=\left(f_{1}(u) g_{1}(v), f_{1}(u) g_{2}(v), f_{2}(u)\right),
$$

which is a spherical product patch [7]. For $\beta(v)=(\cos v, \sin v, 1)$ the surface patch

$$
X(u, v)=\left(f_{1}(u) \cos v, f_{1}(u) \sin v, f_{2}(u)\right),
$$


becomes a surface of revolution [8].

Example 2. The mixed product $\alpha(u)=(u, 1)$ with $\beta(v)=(\cos (v), \sin (v), b v)$ forms the surface patch

$$
X(u, v)=(u \cos (v), u \sin (v), 1)
$$

becomes a helicoid which is a minimal surface in $\mathbb{E}^{3}[8]$.

Example 3. The mixed product $\alpha(u)=(\lambda, u)$ with $\beta(v)=\left(g_{1}(v), g_{2}(v), g_{3}(v)\right)$ forms the surface patch

$$
X(u, v)=\lambda\left(g_{1}(v), g_{2}(v), 0\right)+u\left(0,0, g_{3}(v)\right)
$$

which is a ruled surface. Further, for the given vector $\gamma=\left(0,0, g_{3}(v)\right)$ the cross product $\gamma \times \gamma^{\prime}$ vanishes identically. So the ruled surface given with the patch (13) is cylindrical.

We prove the following results;

Theorem 2. Let $M$ be a mixed product surface given with the patch (8). Then the Gaussian curvature $K$ of $M$ becomes

$$
\begin{aligned}
K=\frac{1}{W^{4}} & \left\{\left(A g_{1} f_{1}^{\prime \prime}+B g_{2} f_{1}^{\prime \prime}+C g_{3} f_{1} f_{1}^{\prime} f_{2}^{\prime \prime}\right)\left(A f_{1} g_{1}^{\prime \prime}+B f_{1} g_{2}^{\prime \prime}+C f_{2} f_{1} f_{1}^{\prime} g_{3}^{\prime \prime}\right)\right. \\
& \left.-\left(A g_{1}^{\prime} f_{1}^{\prime}+B g_{2}^{\prime} f_{1}^{\prime}+C g_{3}^{\prime} f_{1} f_{1}^{\prime} f_{2}^{\prime}\right)^{2}\right\}
\end{aligned}
$$

where

$$
\begin{aligned}
& A=g_{3}^{\prime} f_{2} f_{1}^{\prime} g_{2}-f_{2}^{\prime} g_{3} g_{2}^{\prime} f_{1}, \\
& B=g_{1}^{\prime} g_{3} f_{2}^{\prime} f_{1}-g_{3}^{\prime} g_{1} f_{1}^{\prime} f_{2}, \\
& C=g_{2}^{\prime} g_{1}-g_{1}^{\prime} g_{2},
\end{aligned}
$$

are real valued differentiable functions.

Proof. The tangent space of $M$ is spanned by the vector fields in (9)

$$
\begin{aligned}
& \frac{\partial X}{\partial u}=\left(g_{1} f_{1}^{\prime}, g_{2} f_{1}^{\prime}, g_{3} f_{2}^{\prime}\right), \\
& \frac{\partial X}{\partial u}=\left(f_{1} g_{1}^{\prime}, f_{1} g_{2}^{\prime}, f_{2} g_{3}^{\prime}\right)
\end{aligned}
$$

Hence the coefficients of the first fundamental form of the surface are

$$
\begin{aligned}
& E=\left\langle X_{u}, X_{u}\right\rangle=\left(f_{1}^{\prime}\right)^{2}\left(\left(g_{1}\right)^{2}+\left(g_{2}\right)^{2}\right)+\left(f_{2}^{\prime}\right)^{2}\left(g_{3}\right)^{2}, \\
& F=\left\langle X_{u}, X_{v}\right\rangle=f_{1} f_{1}^{\prime}\left(g_{1} g_{1}^{\prime}+g_{2} g_{2}^{\prime}\right)+f_{2} f_{2}^{\prime} g_{3} g_{3}^{\prime}, \\
& G=\left\langle X_{v}, X_{v}\right\rangle=\left(f_{1}\right)^{2}\left(\left(g_{1}^{\prime}\right)^{2}+\left(g_{2}^{\prime}\right)^{2}\right)+\left(f_{2}\right)^{2}\left(g_{3}^{\prime}\right)^{2},
\end{aligned}
$$

where $\langle$,$\rangle is the standard scalar product in \mathbb{E}^{3}$.

The second partial derivatives of $X(u, v)$ are expressed as follows

$$
\begin{aligned}
& X_{u u}=\left(f_{1}^{\prime \prime} g_{1}, f_{1}^{\prime \prime} g_{2}, f_{2}^{\prime \prime} g_{3}\right), \\
& X_{u v}=\left(f_{1}^{\prime} g_{1}^{\prime}, f_{1}^{\prime} g_{2}^{\prime}, f_{2}^{\prime} g_{3}^{\prime}\right), \\
& X_{v v}=\left(f_{1} g_{1}^{\prime \prime}, f_{1} g_{2}^{\prime \prime}, f_{2} g_{3}^{\prime \prime}\right)
\end{aligned}
$$


Further, the unit normal vector of $M$ is

$$
N=\frac{1}{\sqrt{A^{2}+B^{2}+\left(f_{1}\right)^{2}\left(f_{1}^{\prime}\right)^{2} C^{2}}}\left(A, B, f_{1} f_{1}^{\prime} C\right) .
$$

Using (4), (17) and (18) we can calculate the coefficients of the second fundamental form as follows:

$$
\begin{aligned}
& e=\left\langle X_{u u}, N\right\rangle=\frac{f_{1}^{\prime \prime} g_{1} A+f_{1}^{\prime \prime} g_{2} B+f_{2}^{\prime \prime} g_{3} f_{1} f_{1}^{\prime} C}{\sqrt{A^{2}+B^{2}+\left(f_{1}\right)^{2}\left(f_{1}^{\prime}\right)^{2} C^{2}}}, \\
& f=\left\langle X_{u v}, N\right\rangle=\frac{f_{1}^{\prime} g_{1}^{\prime} A+f_{1}^{\prime} g_{2}^{\prime} B+f_{2}^{\prime} g_{3}^{\prime} f_{1} f_{1}^{\prime} C}{\sqrt{A^{2}+B^{2}+\left(f_{1}\right)^{2}\left(f_{1}^{\prime}\right)^{2} C^{2}}}, \\
& g=\left\langle X_{v v}, N\right\rangle=\frac{f_{1} g_{1}^{\prime \prime} A+f_{1} g_{2}^{\prime \prime} B+f_{2} g_{3}^{\prime \prime} f_{1} f_{1}^{\prime} C}{\sqrt{A^{2}+B^{2}+\left(f_{1}\right)^{2}\left(f_{1}^{\prime}\right)^{2} C^{2}}}
\end{aligned}
$$

Further, substituting (16) and (19) into (6) we get (14).

Theorem 3. Let $M$ a mixed surface given with the patch (8). Then the mean curvature of $M$ becomes

$$
\begin{aligned}
H & =\frac{1}{2 W^{3}}\left\{\left(f_{1}^{\prime \prime} g_{1} A+f_{1}^{\prime \prime} g_{2} B+f_{2}^{\prime \prime} g_{3} f_{1} f_{1}^{\prime} C\right)\left(\left(f_{1}\right)^{2}\left(\left(g_{1}^{\prime}\right)^{2}+\left(g_{2}^{\prime}\right)^{2}\right)+\left(f_{2}\right)^{2}\left(g_{3}^{\prime}\right)^{2}\right)\right. \\
& +\left(f_{1} g_{1}^{\prime \prime} A+f_{1} g_{2}^{\prime \prime} B+f_{2} g_{3}^{\prime \prime} f_{1} f_{1}^{\prime} C\right)\left(\left(f_{1}^{\prime}\right)^{2}\left(\left(g_{1}\right)^{2}+\left(g_{2}\right)^{2}\right)+\left(f_{2}^{\prime}\right)^{2}\left(g_{3}\right)^{2}\right) \\
& \left.-2\left(f_{1}^{\prime} g_{1}^{\prime} A+f_{1}^{\prime} g_{2}^{\prime} B+f_{2}^{\prime} g_{3}^{\prime} f_{1} f_{1}^{\prime} C\right)\left(f_{1} f_{1}^{\prime}\left(g_{1} g_{1}^{\prime}+g_{2} g_{2}^{\prime}\right)+f_{2} f_{2}^{\prime} g_{3} g_{3}^{\prime}\right)\right\} .
\end{aligned}
$$

where $A, B$ and $C$ are real valued differentiable functions defined in (15).

Proof. Using the equations (7), (16) and (19) we get the result.

As a consequence of Theorem 5 we obtain the following result.

Corollary 1 . The mixed product of the straight line $\alpha(u): y(u)=x(u)$ with the space curve $\beta(v)=\left(g_{1}(v), g_{2}(v), g_{3}(v)\right)$ forms the surface patch

$$
X(u, v)=x(u) \beta(v)
$$

is a flat surface, which is a conical surface.

Definition 2. Let $\beta: J \subset \mathbb{R} \longrightarrow \mathbb{E}^{3}$ be a regular curve in $\mathbb{E}^{3}$. If $\langle\beta, \vec{B}\rangle=0$ then $\beta(v)$ is called osculating curve in $\mathbb{E}^{3}$.

Proposition 1. Let $M$ be a mixed product of the straight line $\alpha(u): y(u)=x(u)$ with the unit speed curve $\beta(v)=\left(g_{1}(v), g_{2}(v), g_{3}(v)\right)$. If $\beta(v)$ is an osculating space curve then $M$ is a minimal surface.

Proof. The tangent space of the surface given with the patch (21) is spanned by the vector fields

$$
X_{u}(u, v)=x^{\prime}(u) \beta(v), \quad X_{v}(u, v)=x(u) \beta^{\prime}(v) .
$$

Hence the coefficients of the first fundamental form of the surface are

$$
\begin{aligned}
& E=\left\langle X_{u}, X_{u}\right\rangle=\left(x^{\prime 2}\|\beta(v)\|^{2},\right. \\
& F=\left\langle X_{u}, X_{v}\right\rangle=x(u) x^{\prime}(u) \beta(v) \beta^{\prime}(v), \\
& G=\left\langle X_{v}, X_{v}\right\rangle=(x(u))^{2} \| \beta^{\prime 2} .
\end{aligned}
$$


The second partial derivatives of $X(u, v)$ are expressed as follows

$$
\begin{aligned}
& X_{u u}=x^{\prime \prime}(u) \beta(v), \\
& X_{u v}=x^{\prime}(u) \beta^{\prime}(v), \\
& X_{v v}=x(u) \beta^{\prime \prime}(v) .
\end{aligned}
$$

Further, the unit normal vector of $M$ is

$$
N=\frac{X_{u} \times X_{v}}{\left\|X_{u} \times X_{v}\right\|}=\frac{\beta(v) \times \beta^{\prime}(v)}{\left\|\beta(v) \times \beta^{\prime}(v)\right\|}
$$

Using (4), (22) and (23) we can calculate the coefficients of the second fundamental form as follows:

$$
\begin{aligned}
& e=\left\langle X_{u u}, N\right\rangle=\frac{x^{\prime \prime}(u)}{\left\|\beta(v) \times \beta^{\prime}(v)\right\|}\left\langle\beta(v), \beta(v) \times \beta^{\prime}(v)\right\rangle=0, \\
& f=\left\langle X_{u v}, N\right\rangle=\frac{x^{\prime}(u)}{\left\|\beta(v) \times \beta^{\prime}(v)\right\|}\left\langle\beta^{\prime}(v), \beta(v) \times \beta^{\prime}(v)\right\rangle=0, \\
& g=\left\langle X_{v v}, N\right\rangle=\frac{x(u)}{\left\|\beta(v) \times \beta^{\prime}(v)\right\|}\left\langle\beta^{\prime \prime}(v), \beta(v) \times \beta^{\prime}(v)\right\rangle .
\end{aligned}
$$

Further, substituting (24) into (6) we get the mean curvature of this surface turns into

$$
H=\frac{\kappa\langle\beta, \vec{B}\rangle}{2 x\left\|\beta \times \beta^{\prime}\right\|} .
$$

If $\beta(v)$ is an osculating space curve, i.e., $\langle\beta, \vec{B}\rangle=0$, then the mean curvature $H$ vanishes identically. This completes the proof of the proposition.

\section{Visualization}

I) In the sequel we construct a some 3D geometric shape models by using mixed products given parametrically in the Equation (1). We used maple plotting commands to plot the surface shapes. First, we construct a geometric model of the mixed product of a $2 \mathrm{D}$ curve $\alpha(u)=\left(f_{1}(u), f_{2}(u)\right)$ with a $3 \mathrm{D}$ curve $\beta(v)=\left(g_{1}(v), g_{2}(v), 1\right)$. Actually, these surface are also known as spherical products of two $2 \mathrm{D}$ curves [1]. We consider the following special examples:

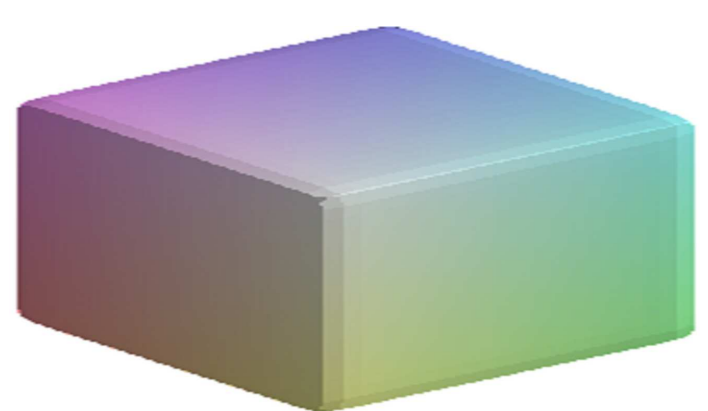

(a)

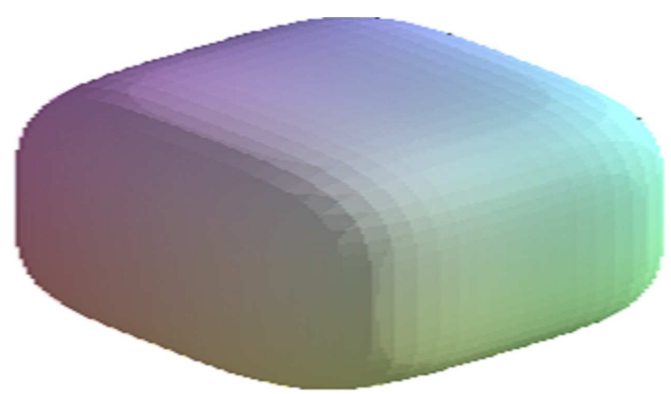

(b)

Fig. 1: (a) $\alpha(u)=\left(\cos ^{0.1}(u), \sin ^{0.1}(u)\right)$ with $\beta(v)=\left(\cos ^{0.1}(v), \sin ^{0.1}(v), 1\right)$. (b) $\alpha(u)=\left(\cos ^{0.5}(u), \sin ^{0.5}(u)\right)$ with $\beta(v)=$ $\left(\cos ^{0.5}(v), \sin ^{0.5}(v), 1\right)$. 


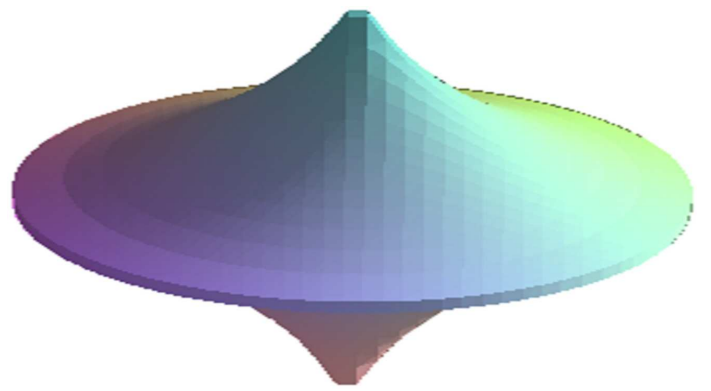

(c)

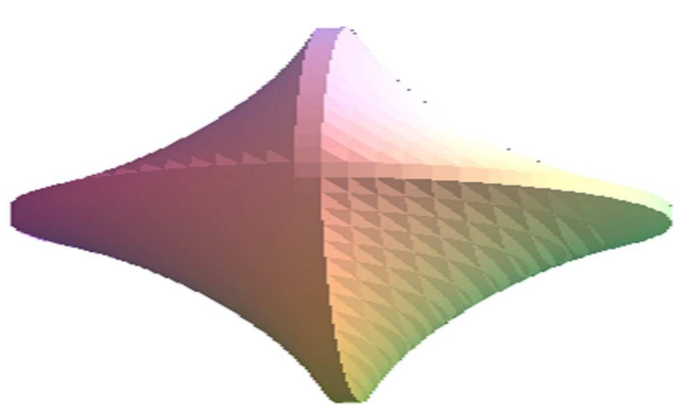

(d)

Fig. 2: (textbf c) $\alpha(u)=\left(\cos ^{3}(u), \sin ^{3}(u)\right)$ with $\beta(v)=(\cos (v), \sin (v), 1)$. (d) $\alpha(u)=(\cos (u), \sin (u))$ with $\beta(v)=$ $\left(\cos ^{3}(v), \sin ^{3}(v), 1\right)$.

II) In the sequel we construct a some 3D geometric shape models by using mixed products given parametrically in the Equation (8):

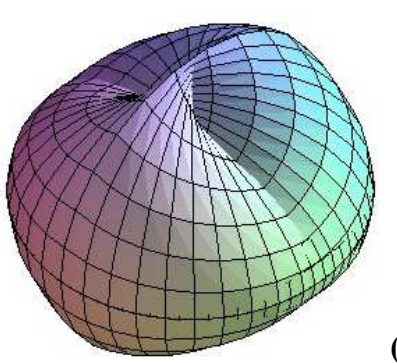

(e)

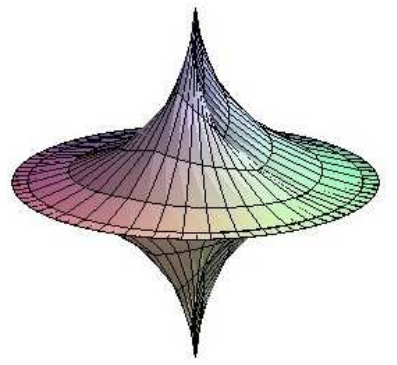

(f)

Fig. 3: (e) The mixed product of $\alpha(u)=(\cos (u), \sin (u))$ with $\beta(v)=(\cos (v), \sin (v), v)$. (f) The mixed product of $\alpha(u)=$ $\left(\cos ^{3}(u), \sin ^{3}(u)\right)$ with $\beta(v)=(\cos (v), \sin (v), v)$.

III) In the sequel we construct a some 3D geometric shape models by using mixed products given parametrically in the Equation (21):

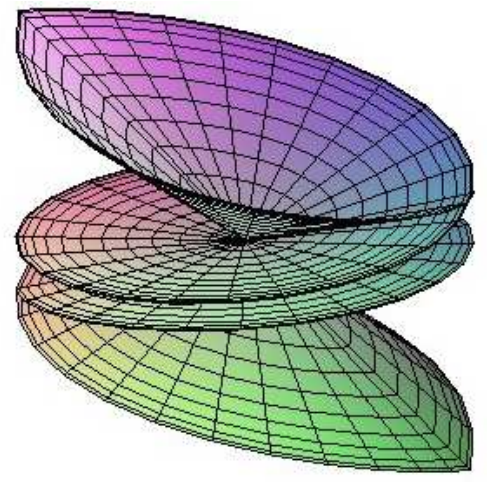

Fig. 4: The mixed product of $\alpha(u): y(u)=x(u)=\cos (u)$ with $\beta(v)=\left(\cos (v), \sin (v), v^{2}\right)$. 


\section{Conclusion}

In this paper, a method of mixed product surface of two a 2D curve with a 3D curve is investigated. Actually, mixed product surfaces are the generalization of the spherical product surfaces. For demonstrating the performance of the proposed method, parameters of superquadrics models were constructed from the spherical products of two super-ellipses. In fact, superquadrics are solid models that can have fairly simple parametrization of representing a large variety of standard geometric solids, as well as smooth shapes in between. This makes them much more convenient for representing rounded, blob-like shape parts, typical for object formed by natural process. By the use of main results of differential geometry we classify the mixed product surfaces of flat or minimal type. Moreover, this frame work can be used for the modelling of some shapes which are more complex than spherical product surfaces. For future work it will be necessary to improve the system to allow for the mixed product surface patch of a 3D curve with a $3 \mathrm{D}$ curve which will be a surface in $\mathbb{E}^{4}$.

\section{References}

[1] Arslan, K., Bulca, B., Bayram, B., Öztürk and Ugail, H., On Spherical Product Surfaces in $\mathbb{E}^{3}$, IEEE Computer Society, International Conference on Cyberworlds, 2009, 132-137.

[2] Chen, B.Y., Geometry of Submanifolds, Dekker, New York, 1973.

[3] Gielis, J., Beirinchx, J. and Bastianens, Superquadrics with rational and irrational symmetry, Symposium on solid modelling and Applications, 2003.

[4] Gupta, A. and Bajcsy, R., Surface and volumetric segmentation of range images using biquadrics and superquadrics. In Int. Conf. Pattern Recognition, 1(1992), 158-162.

[5] Hager, G., Task-directed computation of qualitative decisions from sensor data, IEEE Transactions of Robotics and Automations, 10(1994), 415-429.

[6] Jaklic, A., Leonardis, A. and Solina, F., Segmentation and Recovery of Superquadrics, Kluwer Academic Publishers, $20,2000$.

[7] Leonardis, A., Jaklic, A. and Solina, F., Superquadrics for segmenting and modelling data. Pattern Analysis and Machine Intelligence, IEEE Transactions on, 1997, 19(11): 1289-1295.

[8] O’ Neill, B., Elementary Differential Geometry, Academic Press, USA, 1997.

[9] Pentland, A.P., Percettual Organization and the Representation of Natural Form, Artificial Intelligence, 28(1986), $293-331$.

[10] Pentland, A.P., Automatic Extraction of Deformable Part Models, Int. J. Com. Vision, 4(1990), 107-126. 